\title{
Autism Spectrum, Attachment Styles, and Social Skills in University Student
}

\author{
Junichi Takahashi ${ }^{1}$, Koju Tamaki ${ }^{2}$, Nozomi Yamawaki ${ }^{3}$ \\ ${ }^{1}$ Department of Developmental Disorders, National Institute of Mental Health, \\ National Center of Neurology and Psychiatry, Tokyo, Japan \\ ${ }^{2}$ Department Graduate School of Human Development and Culture, Fukushima University, \\ Fukushima-Shi, Fukushima, Japan \\ ${ }^{3}$ Graduate School of Psychology and Human Development, Nagoya University, Nagoya, Japan \\ Email: j_taka@ncnp.go.jp \\ Received June $6^{\text {th }}, 2013$; revised July $6^{\text {th }}, 2013$; accepted July $13^{\text {th }}, 2013$
}

\begin{abstract}
Copyright (C) 2013 Junichi Takahashi et al. This is an open access article distributed under the Creative Commons Attribution License, which permits unrestricted use, distribution, and reproduction in any medium, provided the original work is properly cited.
\end{abstract}

\begin{abstract}
We investigated the relationships between Autism-spectrum Quotient (AQ) scores, adult attachment style (as assessed by the Internal Working Model [IWM] scale) scores, and social skills (as assessed by Kikuchi's Scale of Social Skills [KiSS-18]) in university students who had no diagnosis of autism spectrum disorder $(N=468)$. The AQ consists of five subscales: social skills, local details, attention switching, communication, and imagination. The IWM is composed of three subscales: secure, anxious, and avoidant attachment styles. The KiSS-18 is a single-factor model. First, we calculated the correlations between AQ, IWM, and KiSS-18 scores. Next, we examined the differences in each subscale score of the IWM between two groups defined by their AQ scores (High and Low AQ groups). We found that the High AQ had higher scores on the IWM secure subscale than did the Low AQ group. In addition, the High AQ group had lower scores on the IWM anxious and avoidant subscales than did the Low AQ group. Moreover, in the High AQ group, the secure style, but not the anxious and avoidant styles, modulated the KiSS-18 scores. The results of the present study add to existing knowledge of the relationships between autism spectrum tendency, adult attachment style, and social skills, and suggested that adult attachment styles (particularly the secure style) may play the role of mediator of social skill ability.
\end{abstract}

Keywords: Autism-Spectrum Quotient; Adult Attachment Style; Social Skill

\section{Introduction}

According to the Diagnostic and Statistical Manual of Mental Disorders (Fourth Edition, Text Revision; DSM-IV-TR, 2000), autism spectrum disorders are developmental disorders characterized by deficits of social interaction, communication, and a restricted range of interests. Those with an autism spectrum disorder frequently exhibit deficits of social skills or social interaction (Laushey \& Heflin, 2000).

In order to examine the deficits of social skills in autism, an approach focusing on attachment problems has been used (Mukaddes, Kaynak, Kinali, Besikci, \& Issever, 2004). Attachment is an affective connection that is typically developed through interactions between children and a mother figure (Bowlby, 1969). Attachment relationships provide an internal representation (internal working model [IWM]) that shows how others interact with the individual. People often derive their sense of self-efficacy from their IWM. Accordingly, positive and negative representations contribute to high and low self-efficacy, respectively. The magnitude of child attachment has been measured by the Strange Situation Procedure (SSP: Ainsworth, Blehar, Waters, \& Wall, 1978). The SSP is an experimental design in which researchers observe children's behavior toward their parent after a short separation. The children are classified into one of three attachment styles: secure, anxious, or avoidant. Children with a secure attachment style do not show resistant behaviors toward their parent, whereas children with an anxious style desire proximity to their parent but simultaneously resist their parent's attempts at affection. Children with an avoidant style display avoidant behaviors and do not seek the proximity of their parent. A previous study (Van IJzendoorn, Goldberg, Kroonenberg, \& Frenk1, 1992) indicated that $65 \%$, $15 \%$, and $20 \%$ of children are generally classified as having a secure, anxious, and avoidant attachment style, respectively.

As for research on the attachment styles of children with autism spectrum disorders, a meta-analysis (Rutgers, BakermansKranenburg, Van IJzendoorn, \& Van Berckelaer-Onnes, 2004) showed that children with autism spectrum disorders exhibit a secure attachment style to the same degree as normally developing children who had no diagnosis of autism spectrum disorder according to the SSP method; specifically, 53\% (range = $40 \%-63 \%$ ) of children with autism were classified having a secure attachment style. According to these results, children with an autism spectrum disorder may show the same development as those without an autism spectrum disorder, at least in terms of attachment formation (Dissanayake \& Crossley, 1997). In contrast, although some studies might propose that the attachment of children with autism spectrum disorder would be 
different from the control children, their results could potentially be due to between-group differences in cognitive ability (e.g., intelligence quotient). Importantly, there were no differences in the attachment styles between these groups of children when the level of cognitive ability was controlled (Rogers, Ozonoff, \& Maslin-Cole, 1991).

A recent study has shown that child attachment theory can be applied to adults throughout the life span (Ainsworth, 1989). In order to measure the magnitude of adult attachment, a previous study (DiTommaso, Brannen-McNulty, Ross, \& Burgess, 2003) developed the Attachment Style Questionnaire. This questionnaire is composed of three short paragraphs that correspond to the three attachment styles from the SSP method (i.e., secure, anxious, and avoidant). Previous studies have examined the relationships between the Attachment Style Questionnaire scores and various psychological adjustments (DiTommaso et al., 2003; Hori \& Kobayashi, 2010; Kanemasa, 2005, 2007; Kanemasa \& Daibo, 2003). This questionnaire has been frequently used to investigate adult attachment and processes good validity and reliability.

The purpose of the current study was to examine the relationships between autism spectrum disorder, attachment style, and social skills in university students who had no formal diagnosis of autism spectrum disorder. Recent studies of autism spectrum disorder have pointed out that there are behavioral and cognitive continuums among people with typical development, Asperger's syndrome, and autism. Moreover, the behavioral and cognitive properties of individuals with autism extend to the general population, as shown by differences in Autismspectrum Quotient (AQ: Baron-Cohen, Wheelwright, Skinner, Martin, \& Clubley, 2001) scores. Thus, by investigating the relationship between AQ scores and Attachment Style Questionnaire scores, we can determine whether the adult attachment styles of people with higher AQ scores (High AQ group) are different from people with lower AQ scores (Low AQ group) in terms of individuals in the general population who had no diagnosis of autism spectrum disorder. Moreover, we aimed to investigate the differences in social skills between the High AQ and Low AQ groups and how adult attachment style influenced this relationship.

\section{Method}

\section{Participants}

The participants were 468 university students (183 men and 285 women). Their ages ranged from 18 to 23 years $(M=19.0$; $S D=1.01)$. They all provided their informed consent before participating. The ethics committee of the Graduate School of Arts and Letters, Tohoku University, approved the study protocol.

\section{Instruments}

We measured autism spectrum tendency (Baron-Cohen et al., 2001) by using the Japanese version of the AQ (Wakabayashi, Baron-Cohen, \& Ashwin, 2012; Wakabayashi, Baron-Cohen, Uchiyama, Yoshida, Tojo, Kuroda, \& Wheelwright, 2007; Wakabayashi, Tojo, Baron-Cohen, \& Wheelwright, 2004; Wakabayashi, Baron-Cohen, \& Wheelwright, 2006). The AQ consists of 50 items referring to various situations. The participants rated each of these items on a 4-point scale. Example items included: "I prefer to do things with others rather than on my own," "I prefer to do things the same way over and over again," and "If I try to imagine something, I find it very easy to create a picture in my mind" (for additional details, see Baron-Cohen et al., 2001). The AQ is composed of five subscales: social skills, local details, attention switching, communication, and imagination. The test-retest reliability and internal consistency of the AQ have been shown to be good (Baron-Cohen et al., 2001). The differences in scores between people with and without an autism diagnosis were similar in the UK and Japan (Wakabayashi et al., 2007). Thus, the results obtained in the UK (BaronCohen et al., 2001) regarding reliability and validity generalizes to the Japanese version of the AQ (Wakabayashi et al., 2006). A high score on the AQ indicates a high degree of autism spectrum traits.

Attachment style was measured using the IWM scale (Takuma \& Toda, 1988). The IWM scale measures adult attachment style in the same way as the Attachment Style Questionnaire (Hazan \& Shaver, 1987) developed by Toda in Japanese. The IWM scale is printed in Japanese, and it consists of 18 items. The participants were asked to rate each of these items on a 5-point scale. Example items on the IWM scale included: "I am easier to get to know than most people," "I have more self-doubts than most people," and "I am more independent and self-sufficient than most people" (for additional details, see the English version Yukawa, Tokuda, \& Sato, 2007). The test-retest reliability and internal consistency of the IWM scale have been shown to be good.

Social skills were measured by Kikuchi's Social Skills Scale (KiSS-18) (Kikuchi, 2004). This questionnaire, printed in Japanese, measures people's skills in receiving a positive reply from others. The KiSS-18 consists of 18 items on various situations involving social interaction. The participants rated these on a 5-point scale. For example, items on the KiSS-18 included the following: "Do you tend to halt a conversation while talking with someone," "Can you explain well what you want someone to do," and "Can you give practical help to others" (for additional details, see the English version Katagami, Ohmura, \& Nitta, 2010). The KiSS-18 is a single-factor model. The test-retest reliability and internal consistency of the KiSS-18 have been shown to be good.

\section{Procedure}

All 468 participants completed the AQ, IWM, and KiSS-18 questionnaires in group settings.

\section{Results}

\section{Psychometric Properties of the AQ, IWM, and KiSS-18 in This Study}

Table 1 shows correlations between scores on the AQ, IWM, and KiSS-18. The mean AQ score was $21.38(S D=6.20)$. The reliability coefficients for social skills, local details, attention switching, communication, and imagination were .69, .56, .37, .50 , and .41 , respectively. We examined the differences between males and females in the total AQ scores. The $t$ test revealed that men had higher AQ scores than women did $[t(466)$ $=2.65, p<.01]$.

The mean IWM score was $63.36(S D=7.89)$. The reliability coefficients for the secure, anxious, and avoidant types were $.86, .79$, and .72 , respectively. There was no significant difference between men and women in the total IWM scores $[t(466)$ 
Table 1.

Correlations between scores on the AQ, IWM, and KiSS-18.

\begin{tabular}{|c|c|c|c|c|c|c|c|c|c|c|c|c|}
\hline & & 1 & 2 & 3 & 4 & 5 & 6 & 7 & 8 & 9 & 10 & 11 \\
\hline 1 & AQ total & 1 & $.73^{* *}$ & $.29^{* *}$ & $.64^{* *}$ & $.70^{* *}$ & $.61^{* *}$ & .10 & $-.44^{* *}$ & $.34^{* *}$ & $.28^{* *}$ & $-.53^{* *}$ \\
\hline 2 & AQ (social skill) & & 1 & $-.14^{* *}$ & $.37^{* *}$ & $.47^{* *}$ & $.39^{* *}$ & -.02 & $-.63^{* *}$ & $.27^{* *}$ & $.36^{* *}$ & $-.57^{* *}$ \\
\hline 3 & AQ (Local details) & & & 1 & .04 & -.10 & -.10 & .11 & $.19^{* *}$ & -.004 & -.02 & $.20^{* *}$ \\
\hline 4 & AQ (Attention switching) & & & & 1 & $.34^{* *}$ & $.24^{* *}$ & .10 & $-.26^{* *}$ & $.26^{* *}$ & $.17^{* *}$ & $-.40^{* *}$ \\
\hline 5 & AQ (Communication) & & & & & 1 & $.34^{* *}$ & .10 & $-.33^{* *}$ & $.35^{* *}$ & $.16^{* *}$ & $-.46^{* *}$ \\
\hline 6 & AQ (Imagination) & & & & & & 1 & .004 & $-.23^{* *}$ & $.14^{* *}$ & .11 & $-.35^{* *}$ \\
\hline 7 & IWM total & & & & & & & 1 & $.35^{* *}$ & $.60^{* *}$ & $.63^{* *}$ & .10 \\
\hline 8 & IWM (Secure) & & & & & & & & 1 & $-.27^{* *}$ & $-.24^{* *}$ & $.66^{* *}$ \\
\hline 9 & IWM (Anxious) & & & & & & & & & 1 & $.26^{* *}$ & $-.37^{* *}$ \\
\hline 10 & IWM (Avoidant) & & & & & & & & & & 1 & $-.18^{* *}$ \\
\hline 11 & KiSS-18 total & & & & & & & & & & & 1 \\
\hline
\end{tabular}

Note: ${ }^{* *} p<.01$.

$=1.23, p=.22]$.

The mean KiSS-18 score was 56.95 (SD = 10.98). The reliability coefficient for the KiSS-18 was .89 . There was no significant difference between males and females in the total KiSS-18 scores $[t(466)=0.40, p=.69]$.

\section{Correlation Analysis}

The AQ (5 subscales: social skill, local details, attention switching, communication, and imagination), the IWM (3 subscales: secure, anxious, and avoidant), and the KiSS-18 (a singlefactor model) were subjected to a correlation analysis. We found significant correlations $(r>.03, p<.01)$ between the total AQ scores and two IWM subscale scores (secure: $r=-.44$, $p<.01$; anxious: $r=.34, p<.01$ ), and between the total AQ scores and KiSS-18 scores $(r=-.53, p<.01)$. We found significant correlations between the social skills subscale scores of the AQ and two IWM subscales scores (secure: $r=-.63, p$ $<.01$; avoidant: $r=.36, p<.01$ ) and between social skills subscale scores and the KiSS-18 scores $(r=-.57, p<.01)$. A significant correlation between attention switching subscale scores of the AQ and the KiSS-18 scores was found $(r=-.40, p<.01)$. There were significant correlations between the communication subscale scores of the AQ and KiSS-18 scores $(r=-.35, p$ $<.01)$.

Moreover, we found significant correlations between secure and anxious subscale scores of the IWM and KiSS-18 scores $(r$ $=.66, p<.01 ; r=-.37, p<.01$, respectively).

\section{Differences in Adult Attachment Style}

In order to examine the characteristics of adult attachment in people with higher AQ score, the participants were divided into groups with high and low AQ scores by adopting a cutoff point proposed by a previous study (Woodbury-Smith, Robinson, Wheelwright, Baron-Cohen, 2005). According to their criterion, people who had an AQ score of greater than or equal to 26 points were classified as having High AQ; those who had AQ scores less than 25 points were classified as Low AQ (High AQ group: $n=121 ; 58$ men and 63 women, $M$ AQ total score $=$
29.30, $S D=2.81$; Low AQ group: $n=347 ; 125$ men and 222 women, $M$ AQ total score $=18.62, S D=4.42]$.

We examined the differences in each IWM subscale (secure, anxious, and avoidant attachment styles) score between the High and Low AQ groups. Regarding the secure attachment style, the High AQ group had lower scores than the Low AQ group $[t(466)=7.74, p<.001]$. For the anxious style, the High AQ group had higher scores than the Low AQ group $[t(466)=$ $5.24, p<.001]$. Finally, for the avoidant style, the High AQ group had higher scores than the Low AQ group $[t(466)=6.30$, $p<.001]$.

\section{Differences in Social Skills}

First, we examined the differences in KiSS-18 scores between the High AQ and Low AQ groups; results showed that the High AQ group had a higher mean score than did the Low AQ group $[t(466)=9.35, p<.001]$.

In order to examine the effects of individual differences, in AQ and IWM results on KiSS-18 scores, participants were divided into groups with high and low IWM secure subscale scores using the median score as a cutoff point. The 32 participants who had a score of exactly 21 points (i.e., the median) were excluded [High IWM secure group: $n=233 ; 78$ men and 155 women, $M$ score $=25.48, S D=2.91$; Low IWM secure group: $n=203$; 92 men and 111 women, $M$ score $=16.63, S D=$ 3.33]. For the anxious subscale, participants were classified into groups with high and low scores based using the median score as a cutoff point. Eleven participants with a score of exactly 23 points (i.e., the median) were excluded [High IWM anxious subscale group: $n=222 ; 90$ men and 132 women, $M$ score $=$ $27.17, S D=2.71$; Low IWM anxious subscale group: $n=210$; 81 men and 129 women, $M$ score $=18.83, S D=2.97]$. Regarding the avoidant subscale, the participants were classified into groups with high and low scores using the median score as a cutoff point. The 14 participants who had a score of exactly 19 points (i.e., the median) were excluded [High IWM avoidant group: $n=209 ; 84$ men and 125 women, $M$ score $=23.22, S D=$ 3.32; Low IWM avoidant group: $n=226$; 91 men and 135 women, $M$ score $=14.93, S D=2.75]$. Next, on the basis of 
these classifications, we created four groups for each of the three IWM subscales: (1) High or Low AQ groups and High or Low IWM secure groups; (2) High or Low AQ groups and High or Low IWM anxious groups; and (3) High or Low AQ groups and High or Low IWM avoidant groups.

Regarding the AQ/IWM secure subscale [High AQ/High IWM: $n=31$; High AQ/Low IWM: $n=87$; Low AQ/High IWM: $n=202$; Low AQ/Low IWM: $n=116$ ], we conducted a one-way analysis of variance (ANOVA) with KiSS-18 score as the between-participants factor of AQ/IWM secure (4 groups; High AQ/High IWM, High AQ/Low IWM, Low AQ/High IWM, Low AQ/Low IWM). We found a significant main effect of the AQ/IWM secure $[F(3,432)=86.194, p<.001]$ (Figure 1(a)). Post-hoc tests (Tukey's HSD method) revealed that the KiSS-18 scores were the highest in Low AQ/High IWM and lowest in the High AQ/Low IWM group [Low AQ/High IWM vs. High AQ/High IWM: $t(432)=3.96, p<.05$; Low AQ/High IWM vs. High AQ/Low IWM: $t(432)=15.10, p<.05$; Low AQ/High IWM vs. Low AQ/Low IWM: $t(432)=10.41, p<.05$; High AQ/Low IWM vs. High AQ/High IWM: $t(432)=5.61, p$ $<.05$; High AQ/Low IWM vs. Low AQ/Low IWM: $t(432)=$ $5.11, p<.05]$. However, there was no difference between the groups of High AQ/High IWM and Low AQ/Low IWM [t(432) $=2.22, p>.05]$.

Regarding the AQ/IWM anxious subscale [High AQ/High IWM: $n=79$, High AQ/Low IWM: $n=35$, Low AQ/High IWM: $n=143$, Low AQ/Low IWM: $n=175$ ], we conducted a one-way ANOVA with KiSS-18 score as the between-participants factor of $\mathrm{AQ} / \mathrm{IWM}$ anxious (4 groups; High $\mathrm{AQ} / \mathrm{High}$ IWM, High AQ/Low IWM, Low AQ/High IWM, Low AQ/ Low IWM). The results showed a significant main effect of the $\mathrm{AQ} / \mathrm{IWM}$ anxious $[F(3,428)=36.01, p<.001]$ (Figure 1(b)). Post-hoc test revealed that the KiSS-18 scores for the Low AQ/Low IWM was the highest compared with other groups [Low AQ/Low IWM vs. High AQ/High IWM: $t(428)=10.03$, $p<.05$; Low AQ/Low IWM vs. High AQ/Low IWM: $t(428)=$ 4.94, $p<.05$; Low AQ/Low IWM vs. High AQ/Low IWM: $t(428)=4.13, p<.05]$. In addition, the Low AQ/High IWM group had higher KiSS-18 scores than the High AQ/Low IWM group $[t(428)=2.38, p<.05]$. However, there was no difference between the High AQ/High IWM and High AQ/Low IWM groups $[t(428)=2.19, p>.05]$.

Regarding the AQ/IWM avoidant subscale [High AQ/High IWM: $n=74$, High AQ/Low IWM: $n=40$, Low AQ/High IWM: $n=135$, Low AQ/Low IWM: $n=186]$, we conducted a one-way ANOVA with KiSS-18 as the between-participants factor of the AQ/IWM anxious subscale (4 groups; High $\mathrm{AQ} /$ High IWM, High AQ/Low IWM, Low AQ/High IWM, Low AQ/Low IWM). The results showed a significant main effect of the AQ/IWM avoidant subscale $[F(3,431)=30.00, p<.001]$ (Figure 1(c)). Post-hoc test revealed that the KiSS-18 score for the Low AQ/Low IWM group was higher than that of the High AQ/High IWM and High AQ/Low IWM [Low AQ/Low IWM vs. High AQ/High IWM: $t(431)=8.27, p<.05$; Low AQ/Low IWM vs. High AQ/Low IWM: $t(428)=5.08, p<.05]$. In addition, the Low AQ/High IWM group had higher KiSS-18 scores than both the High AQ/High IWM and High AQ/Low IWM groups [Low AQ/High IWM vs. High AQ/High IWM: $t(431)=$ $6.65, p<.05$; Low AQ/High IWM vs. High AQ/Low IWM: $t(428)=3.94, p<.05]$.

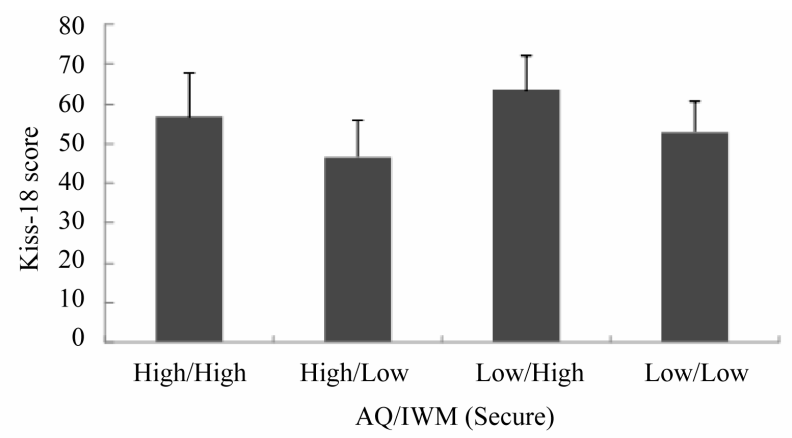

(a)

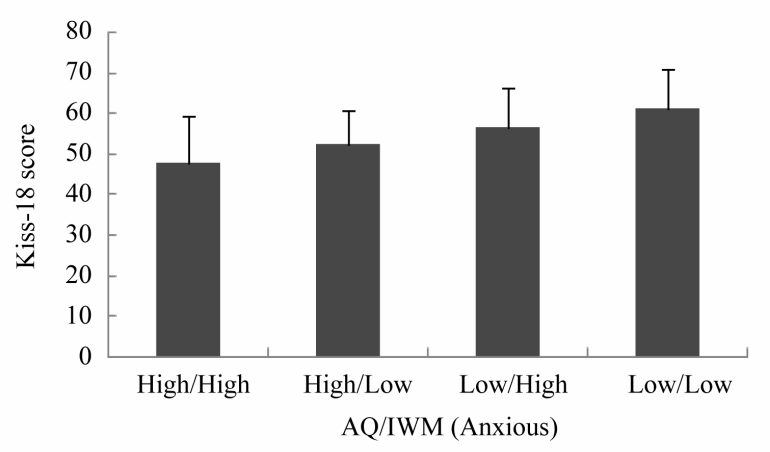

(b)

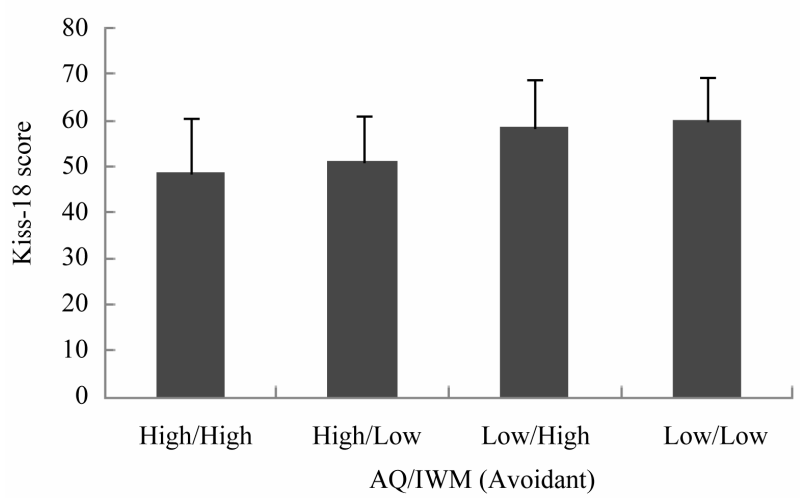

(c)

Figure 1.

Comparisons of the KiSS-18 score on the AQ/IWM. (a) Individual differences in the KiSS-18 scores for the AQ/IWM secure group [High AQ/High IWM: $n=31$; High AQ/Low IWM: $n=87$; Low AQ/High IWM: $n=202$; Low AQ/Low IWM: $n=116$ ]. (b) Individual differences in the KiSS-18 scores for the AQ/IWM anxious group [High AQ/High IWM: $n=79$; High AQ/Low IWM: $n=35$; Low AQ/High IWM: $n=143$; Low AQ/Low IWM: $n=175]$. (c) Individual differences in the KiSS-18 scores for the AQ/IWM secure groups [High AQ/High IWM: $n=74$; High AQ/Low IWM: $n=40$; Low AQ/High IWM: $n=135$; Low AQ/Low IWM: $n=186]$. The error bars denote the standard error of the mean.

\section{Discussion}

Our present study investigated the relationships between the tendency for autism spectrum disorder (indexed by the AQ), adult attachment styles (indexed by the IWM), and social skills (indexed by the KiSS-18) of university students with typical development by using a questionnaire method.

First, we calculated correlations between the total AQ, IWM, and KiSS-18 scores. Specifically, there were significant corre- 
lations between total AQ scores and scores of the IWM secure and anxious subscales. Also, there were small but significant correlations between AQ scores and IWM avoidant subscale scores. These results indicated that the higher the AQ score, the lower the IWM secure subscale score. Moreover, the higher the AQ score, the higher the IWM anxious and avoidant subscales score. In addition, the AQ scores were significantly correlated with the KiSS-18 scores, indicating that the higher the AQ score, the lower the KiSS-18 score.

Furthermore, we studied the characteristics of adult attachment in people with higher AQ scores (High AQ group). The High AQ group had higher IWM secure subscale scores than did the Low AQ group, but lower anxious and avoidant subscale scores. These results indicated that people with High AQ scores might less of a tendency to exhibit secure attachment and a greater tendency to exhibit anxious and avoidant attachment styles.

Moreover, we examined the effects of the interaction of the AQ and IWM on KiSS-18 scores by composing the following 4 groups for each of the three attachment subscale scores: (1) the High AQ/High IWM group, (2) the High AQ/Low IWM group, (3) the Low AQ/High IWM group, and (4) the Low AQ/Low IWM group. Regarding the secure subscale of the IWM, KiSS18 scores for each group were ordered as follows: Low $\mathrm{AQ} /$ High IWM secure $>$ High AQ/High IWM secure = Low AQ/ Low IWM secure $>$ High AQ/Low IWM secure. For the anxious subscale, KiSS-18 scores for each group were ordered as follows: Low AQ/Low IWM anxious > Low AQ/High IWM anxious $>$ High AQ/High IWM anxious = High AQ/Low IWM anxious. Finally, for the avoidant subscale of the IWM, KiSS18 scores for each group were Low AQ/High IWM avoidant = Low AQ/Low IWM avoidant > High AQ/High IWM avoidant $=$ the High $\mathrm{AQ} /$ Low IWM avoidant. These results indicated that people with High AQ scores generally had low KiSS-18 scores. In this regard, when participants had a High IWM secure score, they tended to have a similarly KiSS-18 score compared with people with a Low AQ score or a Low IWM secure score. In contrast, for the anxious attachment style, people with a High AQ score tended to score the lowest on the KiSS-18 compared with other group, regardless of whether they had a High or a Low IWM anxious subscale score. The same tendencies were observed for the avoidant attachment style.

Previous studies have examined the attachment style characteristics of children with autism using the SSP (Ainsworth et al., 1978). The results (Rutgers et al., 2004) showed that 53\% (range $=40 \%-63 \%$ ) of children with autism were classified as having a secure attachment style. Recent studies have shown that child attachment theory can be extended to adults (Ainsworth, 1989) and assessed using questionnaires. In addition, in studying autism spectrum disorder, the behavioral and cognitive characteristics of individuals with autism can extend to individuals with typical development who had no diagnosis by differing on the AQ score (Baron-Cohen et al., 2001). In view of this background, the present study, using a questionnaire survey, showed how adult attachment styles interact with autism spectrum traits for those with no formal diagnosis of autism spectrum disorders. We found that people with higher AQ scores showed a reduced tendency for a secure attachment style and a greater tendency for anxious and avoidant attachment styles. These results are consistent with a previous study (Rutgers, Van IJzendoorn, Bakermans-Kranenburg, Swinkels, Van Daalen, Dietz, Naber, Buitelaar, \& Van Engeland, 2007), which compared Brief Attachment Screening Questionnaire scores among children with autism spectrum, typically developing children, and children with other clinical disorders. They showed that autistic children were far less likely to have a secure style than typically developing children. However, previous studies (Rutgers et al., 2004; Dissanayake \& Crossley, 1997) have also indicated that children with autism spectrum disorders may show normal development in attachment formation, just as in typically developing children. Regarding this point, as in the previous study (Rutgers et al., 2007), we used the questionnaire method and quantitatively analyzed attachment style, as opposed to the qualitative classification of the SSP (Rutgers et al., 2004). Nevertheless, many previous studies have used SSP. We may need to re-examine our results by using other experimental methods such as interview method (Adult Attachment Interview: Main, Kaplan, \& Cassidy, 1985).

The present study also examined the effects of autism spectrum disorder and adult attachment style on social skills. First, the High AQ group showed lower social skills compared with the Low AQ group. This result is the same as was found in previous findings (American Psychiatric Association, DSM-IVTR, 2000; Laushey \& Heflin, 2000). Accordingly, we examined the characteristics of social skills by considering both adult attachment and AQ scores, dividing participants according to their scores on the AQ (High or Low) and their scores for each of the subscales of the IWM (High or Low), creating a total of 12 groups. For the secure subscale, the Low AQ and High IWM group had the highest KiSS-18 score. This result was consistent with the previous studies, indicating that the higher the tendency of autism spectrum disorder, the lower the social skills (Laushey \& Heflin, 2000), while the higher the secure score, the higher the social skills (DiTommaso et al., 2003). For the High AQ groups, the KiSS-18 scores were higher when people had a higher secure tendency than when people had a lower secure tendency. Moreover, there was no significant difference between people with high AQ and high secure scores, and people with low AQ and high secure scores. These results indicated that even if people had a higher tendency of autism spectrum, they had a higher social skill ability when they had a higher tendency of secure style than when they had a lower tendency of secure style, showing a similar level of social skill ability compared to people with lower tendency for AQ and IWM secure when they had a higher tendency for a secure attachment style. For the anxious subscale of the IWM, the Low AQ and Low IWM group had the highest KiSS-18 score. These results were the same as those reported in a previous study (DiTommaso et al., 2003; Laushey \& Heflin, 2000). For the High AQ groups, there was no significant difference in social skills between the High AQ and High IWM anxious group and the High AQ and Low IWM anxious group, indicating that the tendency for autism spectrum disorder mainly affects social skills, regardless of their anxious attachment style tendency. Similar results were observed for the IWM avoidant subscale. Specifically, there was no significant difference in social skills between the High AQ and High IWM avoidant group and the High AQ and Low IWM avoidant group. Considering these results, we speculate that for people with high autism spectrum traits, anxious and avoidant scores did not affect social skills. This is in contrast to the secure style, which could modulate social skill ability. We found that the IWM secure subscale scores were strongly correlated with KiSS-18 scores $(r=.663$, $p<.01)$, whereas he anxious and avoidant subscales were 
weakly correlated with KiSS-18 scores (anxious: $r=-.370, p$ $<.01$; avoidant: $r=-.178, p<.01$ ). We assumed that this finding was due to the weaker correlations between the anxious and avoidant attachment styles and KiSS-18 scores. These attachment styles might not affect the social skill ability of people with a high tendency for $\mathrm{AQ}$.

One might argue that our present results may not be able to be generalized to those with a diagnosis of autism spectrum disorder because our sample was composed of university students who did not have a diagnosis. However, we conducted the present investigation because of the recent suggestion that autistic characteristics of people with a diagnosis may be extended to people that experienced a typical development (Baron-Cohen et al., 2001). In addition, the questionnaire method is useful for investigating the characteristics of adult attachment style (Hazan \& Shaver, 1987). To provide additional support for our present results, future research may need to confirm the present results in people with a diagnosis by using another research method to measure adult attachment such as the interview method or the questionnaire method.

\section{Conclusion}

In conclusion, our present investigation examined the relationships between autism spectrum traits, using a questionnaire method. People with higher autism traits had a higher tendency for a secure attachment style and a lower tendency for anxious and avoidant attachment styles. In addition, we found that in people with a high tendency for autism spectrum disorder, the secure style affected to the social skill ability, whereas the anxious and avoidant styles did not. Our present results provide additional knowledge about the relationships between autism spectrum tendency, adult attachment style, and social skills. Adult attachment style (particularly the secure attachment style) may serve as a mediator in the relationship between autism spectrum traits and social skills.

\section{Acknowledgements}

We would like to thank Dr. Masako Tsurumaki and Dr. Akiko Harano for their valuable support. This work was supported by a Japan Society for the Promotion of Science (JSPS) Grantin-Aid for Scientific Research to J. T. (Grant No. 25-9251).

\section{REFERENCES}

Ainsworth, M. D. S. (1989). Attachments beyond infancy. American Psychologist, 44, 709-716. doi:10.1037/0003-066X.44.4.709

Ainsworth, M. D. S., Blehar, M. C., Waters, E., \& Wall, S. (1978). Patterns of attachment. Hillsdale, NJ: Erlbaum.

American Psychiatric Association (2000). Diagnostic and statistical manual of mental disorders (4th ed.). Washington DC: American Psychiatric Association.

Baron-Cohen, S., Wheelwright, S., Skinner, R., Martin, J., \& Clubley, E. (2001). The Autism-spectrumQuotient (AQ): Evidence from Asperger Syndrome/high-functioning Autism, males and females, scientists and mathematicians. Journal of Autism and Developmental Disorders, 31, 5-17. doi:10.1023/A:1005653411471

Bowlby, J. (1969). Attachment and loss. London: Hogarth Press.

Dissanayake, C., \& Crossley, S. A. (1997). Autistic children's responses to separation and reunion with their mothers. Journal of Autism and Developmental Disorders, 27, 295-312.

doi:10.1023/A:1025802515241

DiTommaso, E., Brannen-McNulty, C., Ross, L., \& Burgess, M. (2003).
Attachment styles, social skills and loneliness in young adults. Personality and Individual Differences, 35, 303-312. doi:10.1016/S0191-8869(02)00190-3

Hazan, C., \& Shaver, P. (1987). Conceptualizing romantic love as an attachment process. Journal of Personality and Social Psychology, 52, 511-524. doi:10.1037/0022-3514.52.3.511

Hori, M., \& Kobayashi, T. (2010). Association among adult attachment, social skills, and psychological adjustment for university students. Journal of School Mental Health, 13, 41-48.

Kanemasa, Y. (2005). Early adult attachment styles, emotional regulation and sensitivity, and interpersonal stress coping: An examination of conceptual consistency in infant and early adult attachment styles. The Japanese Journal of Personality, 14, 1-16. doi:10.2132/personality. 14.1

Kanemasa, Y. (2007). The relationships between early adult attachment styles and adjustment in friendships. The Japanese Journal of Social Psychology, 22, 274-284.

Kanemasa, Y., \& Daibo, I. (2003). Early adult attachment styles and social adjustment. The Japanese Journal of Psychology, 74, 466-473. doi:10.4992/jijpsy.74.466

Katagami, D., Ohmura, H., \& Nitta, K. (2010). Investigation of social adaptive skills by cross-cultural simulation game and KiSS-18. FUZZ-IEEE, 981-986.

Kikuchi, A. (2004). Notes on the researchers using KiSS-18. Bulletin of the Faculty of Social Welfare, Iwate Prefectural University, 6, 41-51.

Laushey, K. M., \& Heflin, J. (2000). Enhancing social skills of kindergarten children with autism through the training of multiple peers as tutors. Journal of Autism and Developmental Disorders, 30, 183-193. doi:10.1023/A:1005558101038

Main, M., Kaplan, N., \& Cassidy, J. (1985). Security in infancy, childhood, and adulthood: A move to the level of representation. Monographs of the Society for Research in Child Development, 50, 66-104. doi: $10.2307 / 3333827$

Mukaddes, N. M., Kaynak, F. N., Kinali, G., Besikci, H., \& Issever, H. (2004). Psychoeducational treatment of children with autism and reactive attachment disorder. Autism, 8, 101-109. doi: $10.1177 / 1362361304040642$

Rogers, S. J., Ozonoff, S., \& Maslin-Cole, C. (1991). A comparative study of attachment behavior in young children with autism or other psychiatric disorders. Journal of the American Academy of child \& Adolescent Psychiatry, 30, 483-488. doi:10.1097/00004583-199105000-00021

Rutgers, A. H., Bakermans-Kranenburg, M. J., Van IJzendoorn, M. H., \& Van Berckelaer-Onnes, I. A. (2004). Autism and attachment: A meta-analytic review. Journal of Child Psychology and Psychiatry, 45, 1123-1134. doi:10.1111/j.1469-7610.2004.t01-1-00305.x

Rutgers, A. H., Van IJzendoorn, M. H., Bakermans-Kranenburg, M. J., Swinkels, S. H. N., Van Daalen, E., Dietz, C., Naber, F. B. A., Buitelaar, J. K., \& Van Engeland, H. (2007). Autism, Attachment and parenting: A comparison of children with autism spectrum disorder, mental retardation, language disorder, and non-clinical children. Journal of Abnormal Psychology, 35, 859-870. doi:10.1007/s10802-007-9139-y

Takuma, T., \& Toda, K. (1988). Adolescent interpersonal attitudes in perspectives of attachment theory: Attempt to create adulthood attachment scales. Journal of Social Sciences and Humanities, 196, 116.

Van IJzendoorn, M. H., Goldberg, S., Kroonenberg, P. M., \& Frenk1, O. J. (1992). The relative effects of maternal and child problems on the quality of attachment: A meta-analysis of attachment in clinical samples. Child Development, 63, 840-858. doi:10.1111/j.1467-8624.1992.tb01665.x

Wakabayashi, A., Baron-Cohen, S., \& Ashwin, C. (2012). Do the traits of autism-spectrum overlap with those of schizophrenia or obsessive-compulsive disorder in the general population? Research in $\mathrm{Au}$ tism Spectrum Disorders, 6, 717-725. doi:10.1016/j.rasd.2011.09.008

Wakabayashi, A., Baron-Cohen, S., Uchiyama, T., Yoshida, Y., Tojo, Y., Kuroda, M., \& Wheelwright, S. (2007). The Autism-spectrum Quotient (AQ) children's version in Japan: A cross-cultural comparison. Journal of Autism and Developmental Disorders, 37, 491-500. doi:10.1007/s10803-006-0181-3 


\section{J. TAKAHASHI ET AL.}

Wakabayashi, A., Tojo, Y., Baron-Cohen, S., \& Wheelwright, S. (2004). The autism-spectrum quotient (AQ) Japanese version: Evidence from high-functioning clinical group and normal adults. The Japanese Journal of Psychology, 75, 78-84. doi:10.4992/jjpsy. 75.78

Wakabayashi, A., Baron-Cohen, S., \& Wheelwright, S. (2006). Are autistic traits an independent personality dimension? A study of the autism-spectrum quotient (AQ) and the NEO-PI-R. Personality and Individual Differences, 41, 873-883. doi:10.1016/j.paid.2006.04.003
Woodbury-Smith, M. R., Robinson, J., Wheelwright, S., \& Baron-Cohen, S. (2005). Screening adults for Asperger's syndrome using the AQ: A preliminary study of its diagnostic validity in clinical practice. Journal of Autism and Developmental Disorders, 35, 331-335. doi:10.1007/s10803-005-3300-7

Yukawa, S., Tokuda, H., \& Sato, J. (2007). Attachment style, self-concealment, and interpersonal distance among Japanese undergraduates. Perceptual and Motor Skills, 104, 1255-1261. 